\title{
Factors Influencing Depression and Anxiety among Black Sexual Minority Men
}

\author{
Louis F. Graham, ${ }^{1}$ Robert E. Aronson, ${ }^{2}$ Tracy Nichols, ${ }^{2}$ \\ Charles F. Stephens, ${ }^{3}$ and Scott D. Rhodes ${ }^{4}$ \\ ${ }^{1}$ School of Public Health, University of Michigan, Ann Arbor, MI 48109-2029, USA \\ ${ }^{2}$ Department of Public Health Education, University of North Carolina Greensboro, Greensboro, NC 27402, USA \\ ${ }^{3}$ Department of Education and Volunteer Services, AID Atlanta, Atlanta, GA 30309, USA \\ ${ }^{4}$ Department of Social Sciences \& Health Policy, Wake Forest University Health Sciences, Winston-Salem, NC 27157, USA
}

Correspondence should be addressed to Louis F. Graham, lfgraham@umich.edu

Received 15 March 2011; Revised 14 June 2011; Accepted 8 July 2011

Academic Editor: John Zeber

Copyright ( $) 2011$ Louis F. Graham et al. This is an open access article distributed under the Creative Commons Attribution License, which permits unrestricted use, distribution, and reproduction in any medium, provided the original work is properly cited.

The primary aim of this study was to examine the relationships between depression and anxiety, and ethnic and sexual identity development, and discrimination and harassment (DH) among Black sexual minority men. Additional aims were to determine whether an interaction effect existed between ethnic and sexual identity and whether coping skills level moderated these relationships. Using an observational cross-sectional design, 54 participants recruited through snowball sampling completed selfadministered online surveys. Stepwise multiple regression analysis was used. Sixty-four percent of the variance in depression scores and $53 \%$ of the variance in anxiety scores were explained by DH and internalized homonegativity together. Thirty percent of the sample had scale scores indicating likelihood of depression and anxiety. Experience of DH and internalized homonegativity explained a large portion of the variability in depression and anxiety among Black sexual minority men. The study showed high prevalence of mental distress among this sample.

\section{Introduction}

Research and theoretical suppositions suggest that Black sexual minority men (BSMM) may experience more depressive symptoms and anxiety than their male heterosexual and Black female counterparts and at minimum parallel those of their white sexual minority counterparts. Little is known about factors that influence the psychosocial health of BSMM. The limited research conducted with BSMM, predominantly White samples of sexual minority men, and Black men with unspecified sexuality, however, indicates that unique concerns related to identity and exposure to violence and discrimination may play important roles. Also critical may be individual's internal and external resources that can serve as coping tools. The primary aim of this study was to examine the relationships between depression and anxiety, and ethnic and sexual identity development (operationalized as Black identity achievement and internalized homonegativity), discrimination and harassment (DH), and coping skills among BSMM.

Mental health disorders affect a considerable proportion of the general population in the United States [1] with roughly $21 \%$ and $29 \%$ of adults meeting Diagnostic and Statistical Manual of Mental Disorders, Fourth Edition criteria for a mood or anxiety disorder, respectively, over their lifetime [2]. General population investigations using random sampling have shown that homo-/bisexual men are more likely than heterosexual men to have mood and anxiety disorders [3-7]. In a meta-analysis, Meyer [8] concluded that the odds of gay and bisexual men experiencing an anxiety or mood disorder over their lifetime were twice those of straight men. In a convenience sample, Cochran and Mays [9] found a $32.6 \%$ prevalence of depression among BSMM. Also, in other studies African American gay men were observed to have an increased probability of feeling anxiety and isolation but were unlikely to seek professional help $[10,11]$. 
Studies show longstanding trends in the high rates of violence, discrimination, and harassment $(\mathrm{VDH})$ perpetuated against sexual minorities [12]. Graham and colleagues' [13] study found a negative effect of violence and discrimination on the mental health status of BSMM. Similarly, Crawford et al. [14] found negative associations between experiences of perceived racist events and life satisfaction and anxiety among BSMM. Finally, in a probability sample of 912 Latino men who had sex with men (MSM), recruited from social venues in New York, Miami, and Los Angeles, $10 \%$ reported that they had experienced violence as an adult because of their sexual orientation or femininity [15]. Other factors influencing mental health outcomes among BSMM may include racial identity development, internalized homonegativity, and coping strategies.

Racial and sexual identity components are core for BSMM [16, 17]. Graham et al. [13] explored the psychosocial health of BSMM and found that BSMM were challenged in developing a healthy identity. They concluded that struggles related to the unique experience of being BSMM such as negative attitudes and beliefs concerning their race and sexuality as well as gender conformity pressure contributed to depression and anxiety. A study by Crawford et al. [14] of African American gay and bisexual men found that BSMM with a positive racial identity were significantly less likely to have mental distress and more likely to have greater life satisfaction and self-esteem than BSMM with less positive racial identity.

Positive racial identity is denoted as "the process of development by which individual members of various socioracial groups overcome the version of internalized racism that typifies their group in order to achieve a selfaffirming and realistic racial-group or collective identity" [18]. According to Helms' [19] model of Black racial identity, the least affirming status is preencounter. The next racial identity phase, encounter, commences once the individual has a personal thought-provoking experience with race that leads the individual to question his racial identity. The third phase, immersion, involves coming to understand the worth and significance of the individual's ethnic heritage. The last stage, internalization, is characterized by affirming and valued perception of oneself. Pierre and Mahalik [20] examined Black racial identity development as a predictor of psychological distress and self-esteem among a college and community sample of 130 Black men of undocumented sexuality aged 18 to 25 years. They found that preencounter and immersion racial identity attitudes were associated with more psychological distress and less self-esteem, while internalization attitudes were associated with greater psychological well-being.

Theoretically, internalized homonegativity hinders the course of healthy identity development [21]. Internalized homonegativity can be described as negative, disapproving, or repudiating views or perceptions of homosexuality or related sexuality components, that persons with a same sex orientation have accepted, believed, or taken on from an external source [22, 23]. Rosser et al. [24] examined the relationship between homosexuality, internalized homonegativity, and mental health in MSM and found that internalized homonegativity, but not homosexuality, was an important predictor of depression in homosexual men.

Coping has been defined as the views and behaviors individuals use to deal with burdens they identify as exceeding their resources [25]. Coping mechanisms include efforts to change the pathway stress takes (problem-focused coping) and efforts to control emotional reactions to stressors (emotion-focused coping). A study by Peterson and colleagues [26] found that psychosocial resources, including optimum social support and spirituality, mediated the effects of stressors on depressive mood among Black MSM. An investigation that included both Black and White homosexual men found that BSMM were more likely to use disengaged coping than White gay men [27]. Other investigations have found a statistical relationship between perceived racism and disengaged coping [28, 29]. Finally, a study of a diverse sample of gay men by David and Knight [30], found that though BSMM were more likely to use disengaged coping styles, they did not appear to experience more negative mental health outcomes as a result.

Few studies have examined the relationships of $\mathrm{DH}$, racial and sexual identity development, and coping with depressive symptoms and anxiety among BSMM. This study therefore examined these relationships. This study focused on the following research questions.

(1) Are internalized homonegativity and DH significantly positively associated with depression and anxiety?

(2) Is Black identity achievement significantly negatively associated with depression and anxiety?

(3) Is there an interaction effect between internalized homonegativity and Black identity achievement on depression and anxiety?

(4) Does coping skill level moderate the associations between depression and anxiety, and internalized homonegativity, DH, and Black identity achievement?

Better understanding mental health determinants among BSMM will enable population health practitioners, medical service providers, and policymakers to help prevent mental distress and disorders, effectively promote overall psychosocial health, and intervene early in distress sequelae.

\section{Methods}

2.1. Design and Sampling. An observational cross-sectional study design was used. The University of North Carolina, Greensboro, served as the primary study site; however, participants were recruited and surveyed at a variety of sites. Volunteer snowball sampling was employed. The study was approved by the university Institutional Review Board (IRB). Posters, flyers, and palm cards were distributed at community facilities and events, and letters were drafted and sent to e-mail and physical address listservs. 
Additionally, advertisements were placed in local newspapers and on social networking websites. The materials included a short description of the study, including its purpose and the contact information of study personnel, as well as a web link for the project. Men 18 years old or over who self-identified as being of African descent (Black, African American, etc.) and as men who had sex with, desired to have sex with, or eroticized sex with men were enrolled in the study. Informed consent was obtained from participants, and they were provided a unique identifier and password to complete a battery of surveys at a secured website accessed from their own personal computer or laptop computer provided for them. Participants were required to answer each question before moving to the next; the battery of surveys took between 30 and 45 minutes to complete, after which participants received a \$25-gift card to Target.

\subsection{Measures}

2.2.1. The Center for Epidemiologic Studies Depression Scale (CES-D). The center for epidemiologic studies depression scale (CES-D) [31] was developed for use with general adult populations (aged 18 or older). This 20 -item self-report scale measures depressive symptoms during the previous 7 days, and responses (0-rarely or none of the time, 3 -most or all of the time) are summed. Sample items include "I felt sad," "I felt lonely," and "I felt fearful." Positive items are reversescored, and the possible range of scores is zero to 60 , with higher scores indicating more symptoms.

Cronbach's alpha for the CES-D in this study was .95. The CES-D may underestimate likelihood of depression given recent evidence indicating that the CES-D may not be an accurate screening tool for ethnic minorities [32]. Depression scores in different racial/ethnic subpopulations might be biased by response patterns that vary between racial groups, not because a community has more or fewer symptoms or disorders, but because the subpopulation articulates psychopathology in a manner not captured by measures normed primarily in a different ethnic group [33]. No better scale has been identified, and given its widespread use among Black and MSM populations, the CES-D was used in this study.

2.2.2. State-Trait Anxiety Inventory (STAI-S). The STAI-S [34], which was used to measure anxiety, is a 20-item questionnaire intended to evaluate current anxiety and has been used with African American populations. Sample items include "I feel calm," "I feel tense," and "I feel upset." The STAI has two factors, anxiety-present and anxiety absent. Anxiety absent items are reverse-scored. Each item is rated from 1 (not at all) to 4 (very much so) to reflect the level of each affect statement, and responses are summed.

Higher scores represent greater anxiety. The STAI-S has demonstrated satisfactory internal consistency and testretest reliability in numerous studies [35]. Additionally, it has demonstrated satisfactory convergent and discriminate validity with other measures $[36,37]$. Cronbach's alpha in this study was .96 .
2.2.3. Demographic Information Sheet. Demographic information sheet asked 10 questions focused on three components of sexuality (orientation, identity, and role), socioeconomic status (educational attainment and annual income), religious affiliation, age, and history of depression diagnosis.

2.2.4. The Black Racial Identity Attitudes Scale (RIAS-B). The black racial identity attitudes scale (RIAS-B) was developed to identify stage placement in the Cross model of minority identity development [38]. The model posits that, as African Americans become aware that they are oppressed, their attitudes toward themselves, their own group, other ethnic minority groups, and members of majority cultures take shape in a way that leads to a central sense of self [39]. The tool consists of 50 statements to which participants are asked to respond using a Likert-type scale (1-strongly disagree, 5strongly agree). Some statements indicate concrete actions, some are descriptive terms, and others are statements of personal values and beliefs. Items reference the current state.

Subscales are scored by averaging items so that the respondent receives a scale score for each of four types of racial identity attitudes (preencounter, encounter, immersion/emersion, and internalization/commitment). The highest mean subscale score reflects placement at that particular racial identity attitude stage. Sample items include "the people I respect most are White," "being Black just feels natural to me," "and White people can't be trusted." Cronbach's alpha for subscales have ranged from .51 to .80 . In this study, Cronbach's alphas were Stage $1-\alpha=.89$, Stage $2-\alpha=.56$, Stage $3-\alpha=.70$, Stage $4-\alpha=.86$.

\subsubsection{Internalized Homonegativity Inventory (IHNI). A 17-} item revised version of the IHNI was used in this study. The IHNI was adapted for use among BSMM because it was originally validated on 241 gay men primarily of European descent [40], it has only been used once among a predominantly Black sample where potential validity issues were raised, and there were men in the current study who identified in diverse ways not referenced in the original IHNI (e.g., bisexual, SGL). Study investigators and a panel of experts assessed translation validity by examining face and content validity of items in the 3 subscales (personal homonegativity, gay affirmation, and morality of homosexuality). Language referencing homosexual and gay was broadened to encompass a wider range of behavior and identity, and two items were added to the personal homonegativity subscale: "I feel ashamed when I see or am around other sexual minority men who are obviously homo/bisexual or who are acting gay/SGL" and "I believe homo/bisexual men are weak."

Principal axis factor analysis with varimax rotation suggested deletion of 8 items (four from the first subscale, three from the second, and one from the third). The original tool and the modified tool were highly correlated with an $r=.96$, which establishes convergent validity in that the tools theoretically should be related to each other. Factor analysis revealed a slightly better performance of the altered IHNI as compared to the original in this sample. The revised 
IHNI factor solution resulted in a decrease of items that crossloaded and appeared slightly more meaningful in that it better reflected the hypothetical factor structure presented in the construct definition of internalized homonegativity.

Sample items include "I am proud to be homo-/bisexual," "I believe that it is morally wrong for men to have sex with other men," and "I sometimes resent my sexual orientation." IHNI items reference current state, responses (1-strongly disagree to 6-strongly agree) are summed, and positive items are reverse-scored. Higher scores on the personal homonegativity and morality of homosexuality subscale and lower scores on the gay affirmation subscale represent more IHNI, where higher scores on the total IHNI represent greater internalized homonegativity. Cronbach's alphas for the adapted 17 item IHNI used in this study were total$\alpha=.97$, Factor $1-\alpha=.95$, Factor $2-\alpha=.91$, Factor $3-$ $\alpha=.89$.

\subsubsection{Perceived Ethnic Discrimination Questionnaire-} Community Version (PEDQ-CV). The PEDQ-CV is used to evaluate perceived ethnic discrimination and was used in this study as a measure of discrimination and harassment $(\mathrm{DH})$. This scale is a modification of the PEDQ-Revised B, developed by Contrada and colleagues [41] to evaluate perceived exposure to discrimination. To develop the community version, the original items were phrased in simpler language and adapted to reflect the everyday experiences of community-dwelling adults [42]. The lifetime discrimination scale (34 items) which includes four subscales (exclusion/rejection, stigmatization, discrimination at work/school, and threat/aggression) and the discrimination in different settings component of the PEDQ-CV were used. Sample items include "people ignored you," "people do not trust you," and "people actually hurt you."

Participants were also asked to indicate whether race, sexuality, both race and sexuality together, or one or the other, but they could not tell which, was primarily involved in their experience of each type of $\mathrm{DH}$ in the discrimination scale and in each community sector in the settings component. The measures are used to assess past-year experiences in social and interpersonal contexts. They have been used with Latino and Black subjects. The past-year discrimination responses (0-never happened to 4-happened daily) are summed, with higher scores representing more DH. Cronbach's alpha in this study was .98 .

2.2.7. Brief COPE. The brief COPE is a shortened adaptation of the COPE Inventory [43]. Comprised of 28 items that measure both active and disengaged coping styles, it includes 14 subscales (of 2 items each) that reflect coping activities. Sample items include "I criticize myself," "I make jokes," and "I learn to live with it." Subscales were combined into two factors: disengaged coping and active coping, consisting of six and eight subscales, respectively; disengaged coping items were reverse-coded.

Active coping includes use of emotional support, use of instrumental support, positive reframing, planning, humor, acceptance, and religion. Disengaged coping includes selfdistraction, denial, substance use, behavioral disengagement, venting, and self-blame. Items ask respondents to consider how they usually feel, think, and respond given stressful or depressing situations or events; response options range from 0 (I usually do not do this at all) to 3 (I usually do this a lot). Cronbach's alpha in this study was .88 .

\section{Results}

3.1. Demographics. Seventy-seven percent of participants indicated that they had sex with or desire to have sex with males only, $77 \%$ self-identified as gay, and $13 \%$ identified culturally as same gender loving (Table 1). Thirty-nine percent had completed a two-year degree or had some college education, and $31 \%$ had completed a four-year degree. The average annual income of participants was $\$ 25,275$, with a range of $\$ 0$ to $\$ 68,000$. The average age was 31 years, with the youngest participant being 19 and the oldest 50;50\% designated their religious affiliation as Christian, and 33\% designated themselves as spiritual. Thirty-three percent of participants said they had been diagnosed with depression by a healthcare professional, 30\% had CES-D scores above 15 indicating likelihood of depression, and 33\% had STAI scores above 39, indicating likelihood of anxiety. Ten percent of participants had CES-D scores and STAI scores indicating both likelihood of depression and anxiety and just $20 \%$ of participants scored into a racial identity development stage lower than internalization.

In the past year 95\% of participants had experienced discrimination and harassment $(\mathrm{DH})$ at least once, and on average $11 \%$ of participants experienced $\mathrm{DH}$ weekly, and 5.3\% experienced $\mathrm{DH}$ daily. Of those experiencing any $\mathrm{DH}$ in the past year, $44 \%$ indicated their race as being primarily involved in the majority of $\mathrm{DH}$ they had experienced in the past year and 32\% indicated both race and sexuality as being primarily involved in the majority of $\mathrm{DH}$ they had experienced (Table 2). In the past year, 52\% of participants had experienced $\mathrm{DH}$ in public places; $43 \%$ had experienced $\mathrm{DH}$ in retail, customer services, or other business settings and 35\% in the criminal justice system. Of those experiencing $\mathrm{DH}$ in public places and retail/customer service, $35 \%$ and $46 \%$, respectively, indicated both their race and sexuality together as being primarily involved and of those experiencing DH in the criminal justice system, $62 \%$ indicated that their race was primarily involved.

3.2. Regression Analysis. Initially, all base variables were entered into a regression model predicting CES-D and STAI scores, with interaction terms added last, in order to determine statistical associations for the full theorized model before beta coefficient significance, strength of dependent and independent variable correlations, and amount of variance accounted for were taken into consideration in specifying the final model. Following this, stepwise independent multiple regression analysis was used to estimate the most parsimonious linear relationship between scores 
TABle 1: Demographics.

\begin{tabular}{lcc}
\hline & $N$ & $\%$ \\
\hline Sexual orientation & 47 & 77 \\
$\quad$ Homosexual & 14 & 23 \\
$\quad$ Bisexual & & \\
Sexual identity & 46 & 77 \\
$\quad$ Gay & 8 & 13 \\
Same-gender-loving & 1 & 2 \\
In the life & 3 & 5 \\
Bisexual & 2 & 3 \\
Other & & \\
Highest level of education & 7 & 12 \\
High school & 24 & 39 \\
Some college/2 yr degree & 19 & 31 \\
4 yr degree & 11 & 18 \\
Terminal degree & & \\
Religious affiliation & 30 & 50 \\
Christian & 20 & 33 \\
Spiritual & 10 & 33 \\
None & 20 & 30 \\
Depression diagnosis & 18 & 33 \\
CES-D Score $>15$ & 20 & \\
STAI Score $>39$ & &
\end{tabular}

$N=61$. Age range $=19-50$ years. Mean age $=30.7$ years.

Annual income range $=0-\$ 68,000$. Mean income $=\$ 25,275$.

on the CES-D and STAI independently and internalized homonegativity, racial identity development, $\mathrm{DH}$, and intersection and interaction terms. To include a proxy measure of racial and sexual identity intersectionality, an internalized homonegativity and racial identity development interaction term was included in the regression equation. The IHNI subscales for this interaction term were reverse coded, such that higher total scores on the IHNI reflect less internalized homonegativity.

It was theorized that those possessing both higher levels of internalized homonegativity and lower levels of positive racial identity would experience greater levels of depression and anxiety than those with lower levels of internalized homonegativity and greater levels of positive racial identity. We recognize that inclusion of this interaction assumes additive identity properties and is not a true measure of intersectional identity, but in absence of any available better quantitative measure authors assessed it necessary to include this proxy term at minimum so as not to ignore the role of intersectional identity. Additionally, research suggests that coping level may moderate the relationships between IHNI, RIAS, IHNI-RIAS, and PEDQ and CES-D and STAI scores; therefore coping and coping-independent variable interaction terms were also included in the regression equation. Internalized homonegativity and Black identity achievement are both rooted in a developmental framework, and therefore age, which is closely related to maturation
TABLE 2: VDH, Identity, and Sector.

\begin{tabular}{lcc}
\hline & $N$ & $\%$ \\
\hline VDH-Implicated Identity & 25 & 44 \\
Race primary & 11 & 19 \\
Sexuality primary & 18 & 32 \\
Race \& Sexuality primary & 3 & 5 \\
Cannot tell which & & \\
VDH-Sector Prevalence & 31 & 52 \\
Public & 26 & 43 \\
Retail & 21 & 35 \\
Criminal justice & 20 & 33 \\
Entertainment Venues & 19 & 32 \\
Religious Institutions & 19 & 32 \\
Workplace/Job & 18 & 30 \\
School/College & 14 & 23 \\
Medical Services & & \\
VDH-Sector-Identity & 11 & 35 \\
Public/Both & 12 & 46 \\
Retail/Both & 13 & 62 \\
Criminal justice/Race & 11 & 55 \\
Entertainment/Both & 11 & 58 \\
Religious/Sexuality & 10 & 53 \\
Workplace/Both & 10 & \\
School/Both & 8 & \\
Medical Services/Both & & \\
\hline
\end{tabular}

Proportion of participants indicating which identity component was primarily involved in the majority of $\mathrm{VDH}$ experienced in the previous year, community sectors with the highest prevalence of $\mathrm{VDH}$, and primary identity component implicated in VDH across sector.

and identity development, was included in the equation as a covariate.

Likewise, a social determinant of health, socioeconomic status, was included in the equation as a covariate. Education and income, which influence resource acquisition, were used to operationalize socioeconomic status. The variables age, education, income, RIAS, IHNI, RIAS-IHNI intersection, PEDQ, coping, and four coping interactions (c-RIAS, cIHNI, c-RIAS-IHNI, c-PEDQ) were selected to test for inclusion in the model (Table 3). Cases with missing data were excluded listwise.

The variables education, income, RIAS, coping, RIASIHNI intersection, and c-RIAS interaction were significantly negatively correlated with CES-D scores $(P<.05)$, and the variables IHNI, PEDQ, and c-PEDQ interaction were significantly positively correlated with CES-D scores $(P<$ $.05)$. The variables age, c-IHNI, and c-RIAS-IHNI were not significantly correlated with CES-D scores. The variables RIAS, coping, and c-RIAS interaction were significantly negatively correlated with STAI scores $(P<.01)$ and the variables IHNI, PEDQ, RIAS-IHNI intersection, and c-PEDQ interaction were significantly positively correlated with STAI scores $(P<.01)$. The variables education, age, income, c-IHNI, and c-RIAS-IHNI were not significantly correlated with STAI scores. 
Table 3: Mean Scores and Standard Deviations of Dependent and Selected Predictor Variables.

\begin{tabular}{lcc}
\hline Analysis Measure & Mean & Std. Dev. \\
\hline CES-D & 13.78 & 12.67 \\
STAI & 38.30 & 13.97 \\
PEDQ & 11.24 & 20.50 \\
IHNI & 30.98 & 17.21 \\
RIAS & 3.67 & .82 \\
Cope & 54.46 & 13.32 \\
\hline
\end{tabular}

$N=54$.

With the base variables education, age, income, RIAS, IHNI, PEDQ, coping, and RIAS-IHNI entered into regression models (CESD, $F=11.73, P<.01$; STAI, $F=7.38$, $P<.01)$, the beta coefficients for IHNI and PEDQ were significant $(P<.05)$ for both models predicting CES-D and STAI scores. Every one unit increase in the IHNI accounted for a .63 increase in CES-D scores and a .7 increase in STAI scores, and every one unit increase in PEDQ accounted for a .23 increase in CES-D scores and a .25 increase in STAI scores. After the interaction terms were added to the models (CESD, $F=10.79, P<.01$; STAI, $F=7.06, P<.01$ ), the beta coefficients for the IHNI and RIAS-IHNI intersection were significant $(P<.05)$ for both models predicting CES$D$ and STAI scores. Every one unit increase in the IHNI corresponded to a 1.97 increase in CES-D scores and a 3.47 increase in STAI scores, and every one unit increase in the RIAS-IHNI intersection corresponded to a .71 decrease in CES-D scores and a .94 decrease in STAI scores.

The PEDQ beta coefficient became nonsignificant, and the RIAS beta coefficient remained nonsignificant for both models predicting CES-D and STAI scores even though the RIAS-IHNI intersection beta coefficient was significant. This suggests that the IHNI variable was driving the significance of the RIAS-IHNI intersection beta coefficient. When the RIAS, IHNI, and the RIAS-IHNI intersection were entered into models, both the RIAS and the RIAS-IHNI intersection beta coefficients became nonsignificant for both models predicting CES-D and STAI scores (not shown). Only the IHNI beta coefficient remained significant.

Using stepwise multiple regression analysis the variables PEDQ and IHNI were entered into the final model for both the CES-D and STAI while age, education, income, RIAS, the RIAS-IHNI intersection, coping, and the four coping interaction terms (c-RIAS, c-RIAS-IHNI, c-IHNI, c-PEDQ) were excluded (Table 4). Taking into account the number of variables in the model and the number of observations, 64\% of the variance in CES-D scores and 53\% of the variance in STAI scores were explained by PEDQ and IHNI together. PEDQ alone accounted for $51 \%$ of the variance in CES-D scores, with IHNI accounting for an additional 13\%. IHNI alone accounted for $46 \%$ of the variance in STAI scores, with PEDQ accounting for an additional 7\%.

The overall models were significant (CES-D, $F=47.89$, $P<.001$; STAI, $F=31.10, P<.001)$; there was a linear relationship between PEDQ and IHNI and CES-D and STAI scores. Holding IHNI constant, for every 1 unit increase in
TABLE 4: Summary of Stepwise Multiple Linear Regression Analysis.

\begin{tabular}{lcccccccc}
\hline $\begin{array}{l}\text { Predictor } \\
\text { Variable }\end{array}$ & Step & $R$ & Adj. $R^{2}$ & $\Delta R^{2}$ & Sig. $\Delta R^{2}$ & $\beta$ & $\mathrm{T}$ & $P$ \\
\hline $\begin{array}{l}\text { Depression } \\
\quad\end{array}$ & & & & & & & \\
$\quad$ PEDQ & 1 & .72 & .51 & & & .47 & 4.67 & $<.001$ \\
$\quad$ IHNI & 2 & .81 & .64 & .13 & $<.001$ & .45 & 4.44 & $<.001$ \\
Anxiety & & & & & & & & \\
$\quad$ IHNI & 1 & .47 & .46 & & & .49 & 4.30 & $<.001$ \\
PEDQ & 2 & .55 & .53 & .07 & .004 & .34 & 3.01 & .004 \\
\hline
\end{tabular}

PEDQ: Perceived Ethnic Discrimination Questionnaire; IHNI: Internalized Homonegativity Inventory. The Beta listed is the standardized value.

PEDQ, CES-D scores increased by .29, and holding PEDQ constant, for every 1 unit increase in IHNI, CES-D scores increased by .33 . Holding PEDQ constant, for every 1 unit increase in IHNI, STAI scores increased by .41, and holding IHNI constant, for every 1 unit increase in PEDQ, STAI scores increased by 24 .

\section{Discussion}

Experience of $\mathrm{DH}$ and internalized homonegativity explained a large portion of the variance in depression and anxiety among this sample, as in other studies [1315, 21, 24, 44-47]. Though experience of DH explained more of the variance in depression than internalized homonegativity and the reverse was true for anxiety, both $\mathrm{DH}$ and internalized homonegativity were very strongly associated with both depression and anxiety. A high percentage of the sample screened positive for likelihood of both depression (30\%) and anxiety (33\%), far higher percentages than in the general population (estimated between $9.3-21 \%$ for depression and $11-29 \%$ for anxiety) $[1,2]$ and higher than the $22 \%$ found for depression and comparable to the $36.7 \%$ found for anxiety among Black gay, lesbian, and bisexual respondents by Meyer et al. [48] and the $32.6 \%$ found for depression by Cochran and Mays [9]. The average CES-D score was 13.78 and the average STAI score was 38.30 , which are roughly equal to the 13.96 and 37.5, respectively, found among a sample of similar aged BSMM in the study by David and Knight [30].

Discrimination and harassment appeared to be chronic among participants in the current study. In the past year, they reported experiencing more $\mathrm{DH}$ and experiencing $\mathrm{DH}$ more often than reported in samples of predominantly White sexual minority men (which range from $3.7 \%$ to $76 \%$ ) [7, $12,49]$. Race independently and race and sexuality together were implicated most by participants as driving factors in their experience of $\mathrm{DH}$. This may be further evidence of the essential role of intersectionality in understanding and contextualizing the relationship between $\mathrm{DH}$ and mental health outcomes among BSMM.

Participants experienced DH most often in public places, retail settings, and the criminal justice system. This finding differs somewhat from the original PEDQ-CV validation study, in which settings with the most reported DH included 
public places, work, and school. The differences, however, may be a result of the inclusion of Latino and women subgroups in the validation study since their experiences may differ from those of BSMM. Most participants in this study indicated both race and sexuality together as being primarily involved in their experiences of $\mathrm{DH}$ in most community settings, except in the criminal justice system, where participants cited race most often, and in religious institutions, where participants cited sexuality most often. Racial identity development did not appear to play a significant role in depression and anxiety in this sample, though the lack of variability in stage placement of participants may in part explain this.

An overwhelming majority of participants fell in the immersion and internalization stages, while very few participants were in the preencounter or encounter stages. Perhaps consequently, neither the racial identity development, identity development intersection, nor their coping interaction measures made it into the final models. Given the moderately strong univariate associations between both depression and anxiety and the race and sexual identity intersection measure, and the significant RIAS-IHNI intersection beta coefficient when all variables were entered into a model without forcing any variables to drop, possibly a different or better measure of Black identity development would have produced different results when forcing variables with nonsignificant coefficients to drop from the model. It appears that either the sample distribution was skewed on stage placement or the measure was not able to adequately differentiate participants across the stages. However, if racial identity development does not in fact play a major role in explaining depression or anxiety outcomes among BSMM, this finding does not support previous findings in the literature on racial identity development as a predictor of mental health outcomes among Black men with undocumented sexuality $[20,50]$.

Level of positive coping was not a significant indicator of depression and anxiety in this sample and thus neither confirm findings among samples of predominately White gay men [51] nor the study conducted by Peterson et al. [26]. This finding is similar to that of David and Knight [30]. In light of these findings, we share David and Knight's [30] conclusions that perhaps resiliency may be a more important mitigating factor for depression and anxiety than positive coping. Age and income were excluded variables that were close to being included in the final model for depression, and age and Black identity achievement variables were close to being included in the final model for anxiety. Perhaps with a larger sample size, and thus greater power, the relationship between depression and age and income, and anxiety and age and Black identity would be significant.

Additional limitations in this study include the use of snowball sampling, which can produce samples that may not be accurate reflections of the target population, and thus the results may not be indicative of the actual trends within the target population. Also, given the small sample size coupled with stepwise regression analysis and a high number of independent variables for possible model inclusion, power is low, $R$ squared values may be overestimated, and confidence intervals for effects and predicted values may be overly narrow. Further research should focus on qualitative and quantitative exploration of the causes of VDH in community settings in which VDH is most prevalent, development and validation of tools for use among BSMM, and further examination of identity development among BSMM, including the influence of spirituality and religious institutions.

The findings of this study suggest that efforts should be increased to implement antidiscriminatory policies in the community settings where VDH is most prevalent, public health practitioners should work to decrease negative attitudes and beliefs regarding ethnic and sexual minority identities, and service providers should help clients alleviate their internalized homonegativity and avoid VDH. Additional implications of findings for treatment and public health initiatives include training clinicians on signs and symptoms of depression and anxiety among BSMM, expansion of mental health services for this subpopulation, and social marketing campaigns that target internalized homonegativity. This inquiry sought to produce scientific evidence that could inform health and quality of life promotion related to VDH and identity development. Findings of this study further develop the conceptual framework of mental disorder acquisition by BSMM.

\section{References}

[1] B. F. Grant, F. S. Stinson, D. A. Dawson et al., "Prevalence and co-occurrence of substance use disorders and independent mood and anxiety disorders: results from the national epidemiologic survey on alcohol and related conditions," Archives of General Psychiatry, vol. 61, no. 8, pp. 807-816, 2004.

[2] R. C. Kessler, P. Berglund, O. Demler, R. Jin, K. R. Merikangas, and E. E. Walters, "Lifetime prevalence and age-of-onset distributions of DSM-IV disorders in the national comorbidity survey replication," Archives of General Psychiatry, vol. 62, no. 6, pp. 593-602, 2005.

[3] S. D. Cochran, C. Keenan, C. Schober, and V. M. Mays, "Estimates of alcohol use and clinical treatment needs among homosexually active men and women in the U.S. population," Journal of Consulting and Clinical Psychology, vol. 68, no. 6, pp. 1062-1071, 2000.

[4] S. D. Cochran and V. M. Mays, "Lifetime prevalence of suicide symptoms and affective disorders among men reporting samesex sexual partners: results from NHANES III," American Journal of Public Health, vol. 90, no. 4, pp. 573-578, 2000.

[5] S. D. Cochran and V. M. Mays, "Relation between psychiatric syndromes and behaviorally defined sexual orientation in a sample of the US population," American Journal of Epidemiology, vol. 151, no. 5, pp. 516-523, 2000.

[6] T. G. M. Sandfort, R. de Graaf, R. V. Bijl, and P. Schnabel, "Same-sex sexual behavior and psychiatric disorders: findings from the Netherlands mental health survey and incidence study (NEMESIS)," Archives of General Psychiatry, vol. 58, no. 1, pp. 85-91, 2001.

[7] V. M. Mays and S. D. Cochran, "Mental health correlates of perceived discrimination among lesbian, gay, and bisexual adults in the United States," American Journal of Public Health, vol. 91, no. 11, pp. 1869-1876, 2001.

[8] I. H. Meyer, "Prejudice, social stress, and mental health in lesbian, gay, and bisexual populations: conceptual issues and 
research evidence," Psychological Bulletin, vol. 129, no. 5, pp. 674-697, 2003.

[9] S. D. Cochran and V. M. Mays, "Depressive distress among homosexually active African American men and women," American Journal of Psychiatry, vol. 151, no. 4, pp. 524-529, 1994.

[10] A. Bell and M. Weinberg, Homosexualities: A Study of Human Diversity among Men and Women, Simon \& Schuster, New York, NY, USA, 1978.

[11] R. Bass-Hass, "The lesbian dyad: basic issues and value systems," Journal of Sex Research, vol. 4, p. 126, 1968.

[12] G. M. Herek, "Hate crimes and stigma-related experiences among sexual minority adults in the united states: prevalence estimates from a national probability sample," Journal of Interpersonal Violence, vol. 24, no. 1, pp. 54-74, 2009.

[13] L. F. Graham, K. Braithwaite, P. Spikes, C. F. Stephens, and U. F. Edu, "Exploring the mental health of black men who have sex with men," Community Mental Health Journal, vol. 45, no. 4, pp. 272-284, 2009.

[14] I. Crawford, K. W. Allison, B. D. Zamboni, and T. Soto, "The influence of dual-identity development on the psychosocial functioning of African-American gay and bisexual men," Journal of Sex Research, vol. 39, no. 3, pp. 179-189, 2002.

[15] R. M. Díaz, G. Ayala, E. Bein, J. Henne, and B. V. Marin, “The impact of homophobia, poverty, and racism on the mental health of gay and bisexual Latino men: findings from 3 US cities," American Journal of Public Health, vol. 91, no. 6, pp. 927-932, 2001.

[16] E. Acosta, "Affinity for Black heritage: seeking life-style within a community," The Washington Blade, pp. A-1-A-25, 1979.

[17] V. M. Mays, S. D. Cochran, and S. Rhue, "The impact of perceived discrimination on the intimate relationships of Black lesbians," Journal of Homosexuality, vol. 25, no. 4, pp. 1-14, 1993.

[18] J. E. Helms and D. A. Cook, Using race and culture in counseling and psychotherapy: theory and process, Allyn \& Bacon, Boston, Mass, USA, 1999.

[19] J. E. Helms, “An update of Helms' White and people of color racial identity development models," in Handbook of Multicultural Counseling, J. G. Ponterotto, J. M. Cases, L. A. Suzuki, and C. M. Alexander, Eds., pp. 188-189, Sage, Thousand Oaks, Calif, USA, 1995.

[20] M. R. Pierre and J. R. Mahalik, "Examining African selfconsciousness and Black racial identity as predictors of Black men's psychological well-being," Cultural Diversity and Ethnic Minority Psychology, vol. 11, no. 1, pp. 28-40, 2005.

[21] A. Malyon, "Psychotherapeutic implications of internalized homophobia in gay men," Journal of Homosexuality, vol. 7, no. 2-3, pp. 59-69, 1982.

[22] W. W. Hudson and W. A. Ricketts, "A strategy for the measurement of homophobia," Journal of Homosexuality, vol. 5, no. 4, pp. 357-372, 1980.

[23] G. Weinberg, Society and the healthy homosexual, Anchor Books, New York, NY, USA, 1973.

[24] B. R. Rosser, W. O. Bockting, M. W. Ross, M. H. Miner, and E. Coleman, "The relationship between homosexuality, internalized homo-negativity, and mental health in men who have sex with men," Journal of Homosexuality, vol. 55, no. 2, pp. 185-203, 2008.

[25] R. S. Lazarus and S. Folkman, Stress, appraisal, and coping, Springer, New York, NY, USA, 1984.

[26] J. L. Peterson, S. Folkman, and R. Bakeman, "Stress, coping, HIV status, psychosocial resources, and depressive mood in African American gay, bisexual, and heterosexual men,"
American Journal of Community Psychology, vol. 24, no. 4, pp. 461-487, 1996.

[27] R. A. Hayes, "Differences in social support, coping, and attitudes toward homosexuality between African-American and Caucasian men living with HIV," Dissertation Abstracts International: Section B: The Sciences and Engineering, vol. 56, no. 9-B, p. 5171, 1996.

[28] R. Clark, "Skin tone, coping, and cardiovascular responses to ethnically-relevant stimuli," Dissertation Abstracts International: Section B: The Sciences \& Engineering, vol. 57, no. 12-B, p. 7719, 1997.

[29] D. I. Lopez-Kinney, "Racism-related stress, appraisal, and coping among Latino/as," Dissertation Abstracts International: Section B: The Sciences \& Engineering, vol. 62, no. 7-B, p. 3383, 2002.

[30] S. David and B. G. Knight, "Stress and coping among gay men: age and ethnic differences," Psychology and Aging, vol. 23, no. 1, pp. 62-69, 2008.

[31] L. S. Radloff, "The CES-D Scale: a self-report depression scale for research in the general population," in Applied Psychological Measurement, pp. 85-401, West Publishing, New York, NY, USA, 1977.

[32] K. M. Perreira, N. Deeb-Sossa, K. M. Harris, and K. Bollen, "What are we measuring? An evaluation of the CES-D across race/ethnicity and immigrant generation," Social Forces, vol. 83, no. 4, pp. 1567-1602, 2005.

[33] W. A. Vega and R. G. Rumbaut, "Ethnic minorities and mental health," Annual Review of Sociology, vol. 17, pp. 351-383, 1991.

[34] C. D. Spielberger, Manual for the State-Trait Anxiety Inventory (Form y), Mind Garden, Palo Alto, Calif, USA, 1983.

[35] L. L. B. Barnes, D. Harp, and W. S. Jung, "Reliability generalization of scores on the spielberger state-trait anxiety inventory," Educational and Psychological Measurement, vol. 62, no. 4, pp. 603-618, 2002.

[36] P. J. Bieling, M. M. Antony, and R. P. Swinson, "The statetrait anxiety inventory, trait version: structure and content reexamined," Behaviour Research and Therapy, vol. 36, no. 7-8, pp. 777-788, 1998.

[37] H. Caci, F. J. Baylé, C. Dossios, P. Robert, and P. Boyer, "The Spielberger trait anxiety inventory measures more than anxiety," European Psychiatry, vol. 18, no. 8, pp. 394-400, 2003.

[38] J. E. Helms and T. A. Parham, "Black racial identity attitude scale (Form RIAS-B)," in Black and White Racial Identity: Theory, Research, and Practice, J. E. Helms, Ed., pp. 167-174, Cobb \& Henry, Hampton, Va, USA, 1990.

[39] W. E. Cross, The Psychology of Nigrescenece: Revising the Cross Model, Sage Publishing, Thousand Oaks, Calif, USA, 1995.

[40] W. Mayfield, "The development of an internalized homonegativity inventory for gay men," Journal of Homosexuality, vol. 41, no. 2, pp. 53-76, 2001.

[41] R. J. Contrada, R. D. Ashmore, M. L. Gary et al., "Measures of ethnicity-related stress: psychometric properties, ethnic group differences, and associations with well-being," Journal of Applied Social Psychology, vol. 31, no. 9, pp. 1775-1820, 2001.

[42] E. Brondolo, K. P. Kelly, V. Coakley et al., "The perceived ethnic discrimination questionnaire: development and preliminary validation of a community version," Journal of Applied Social Psychology, vol. 35, no. 2, pp. 335-365, 2005.

[43] C. S. Carver, "You want to measure coping but your protocol's too long: consider the brief COPE," International Journal of Behavioral Medicine, vol. 4, no. 1, pp. 92-100, 1997.

[44] T. N. Brown, D. R. Williams, J. S. Jackson et al., "Being black and feeling blue: the mental health consequences of racial 
discrimination," Race and Society, vol. 2, no. 2, pp. 117-131, 2000.

[45] S. Karlsen and J. Y. Nazroo, "Relation between racial discrimination, social class, and health among ethnic minority groups," American Journal of Public Health, vol. 92, no. 4, pp. 624-631, 2002.

[46] R. C. Kessler, K. D. Mickelson, and D. R. Williams, "The prevalence, distribution, and mental health correlates of perceived discrimination in the United States," Journal of Health and Social Behavior, vol. 40, no. 3, pp. 208-230, 1999.

[47] K. Siefert, P. J. Bowman, C. M. Heflin, S. Danziger, and D. R. Williams, "Social and environmental predictors of maternal depression in current and recent welfare recipients," American Journal of Orthopsychiatry, vol. 70, no. 4, pp. 510-522, 2000.

[48] I. H. Meyer, J. Dietrich, and S. Schwartz, "Lifetime prevalence of mental disorders and suicide attempts in diverse lesbian, gay, and bisexual populations," American Journal of Public Health, vol. 98, no. 6, pp. 1001-1006, 2008.

[49] D. M. Huebner, G. M. Rebchook, and S. M. Kegeles, "Experiences of harassment, discrimination, and physical violence among young gay and bisexual men," American Journal of Public Health, vol. 94, no. 7, pp. 1200-1203, 2004.

[50] C. T. Pyant and B. J. Yanico, "Relationship of racial identity and gender-role attitudes to Black women's psychological wellbeing," Journal of Counseling Psychology, vol. 38, no. 3, pp. 315-322, 1991.

[51] J. Miranda and M. Storms, "Psychological adjustment of lesbians and gay men," Journal of Counseling \& Development, vol. 68, pp. 41-45, 1989. 


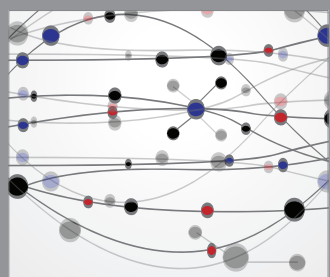

The Scientific World Journal
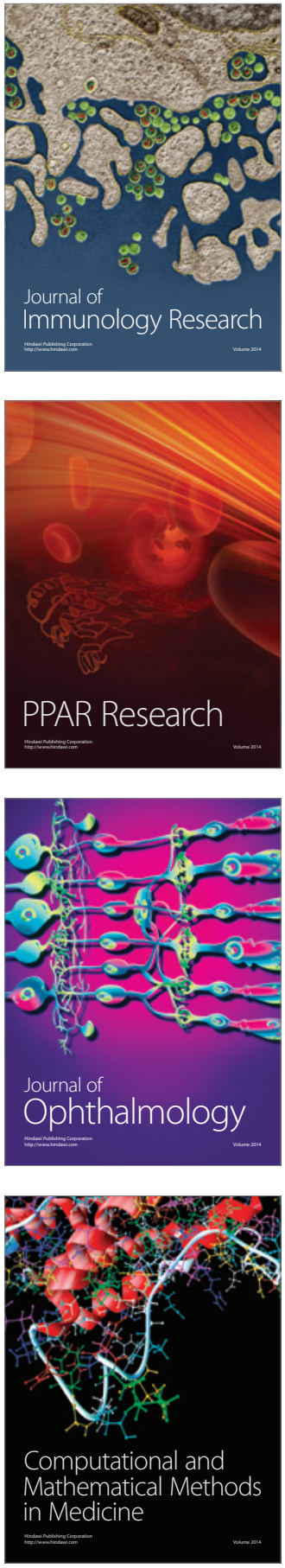

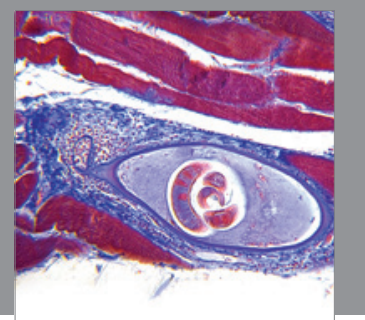

Gastroenterology

Research and Practice
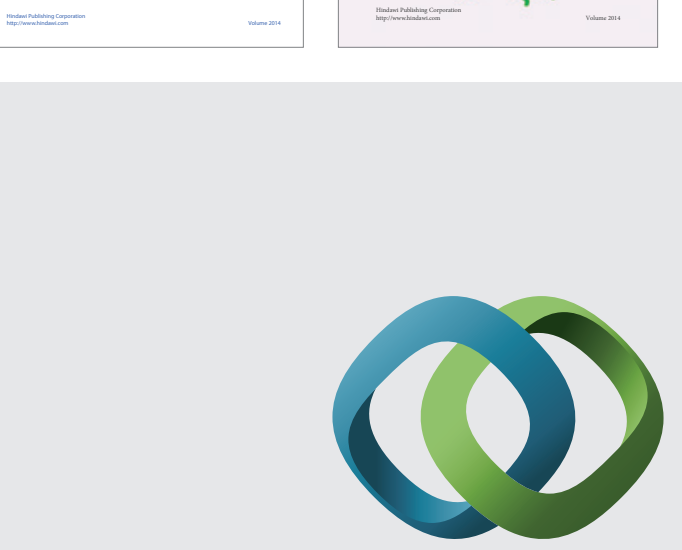

\section{Hindawi}

Submit your manuscripts at

http://www.hindawi.com
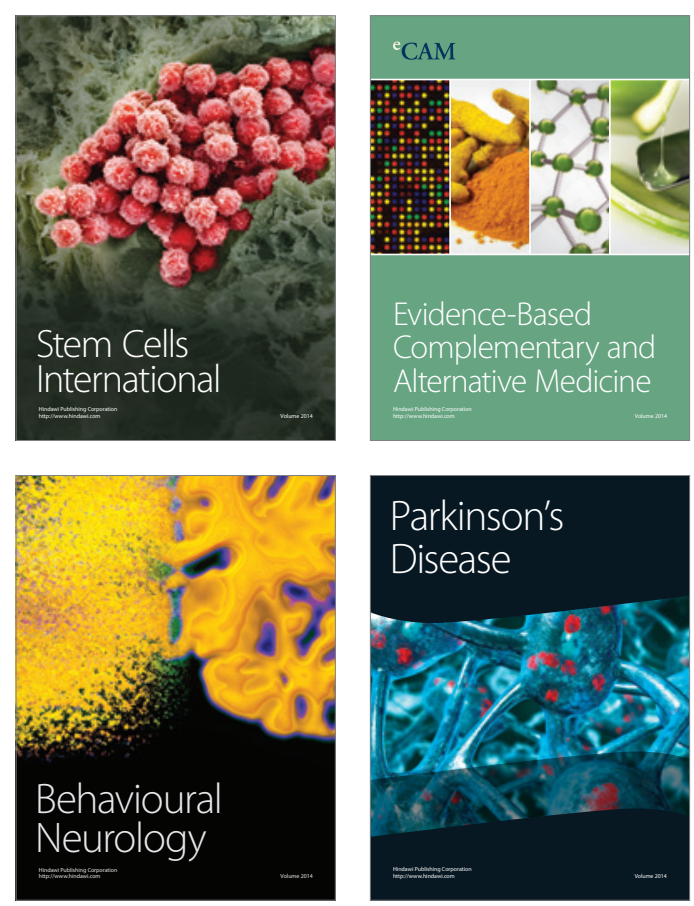

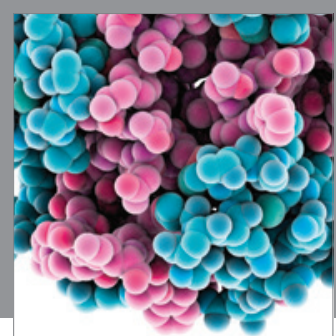

Journal of
Diabetes Research

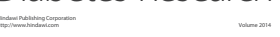

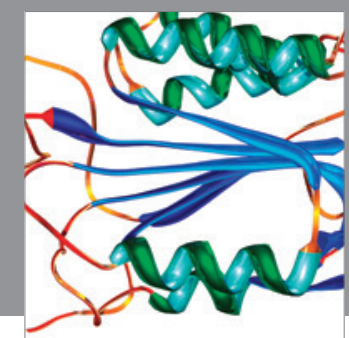

Disease Markers
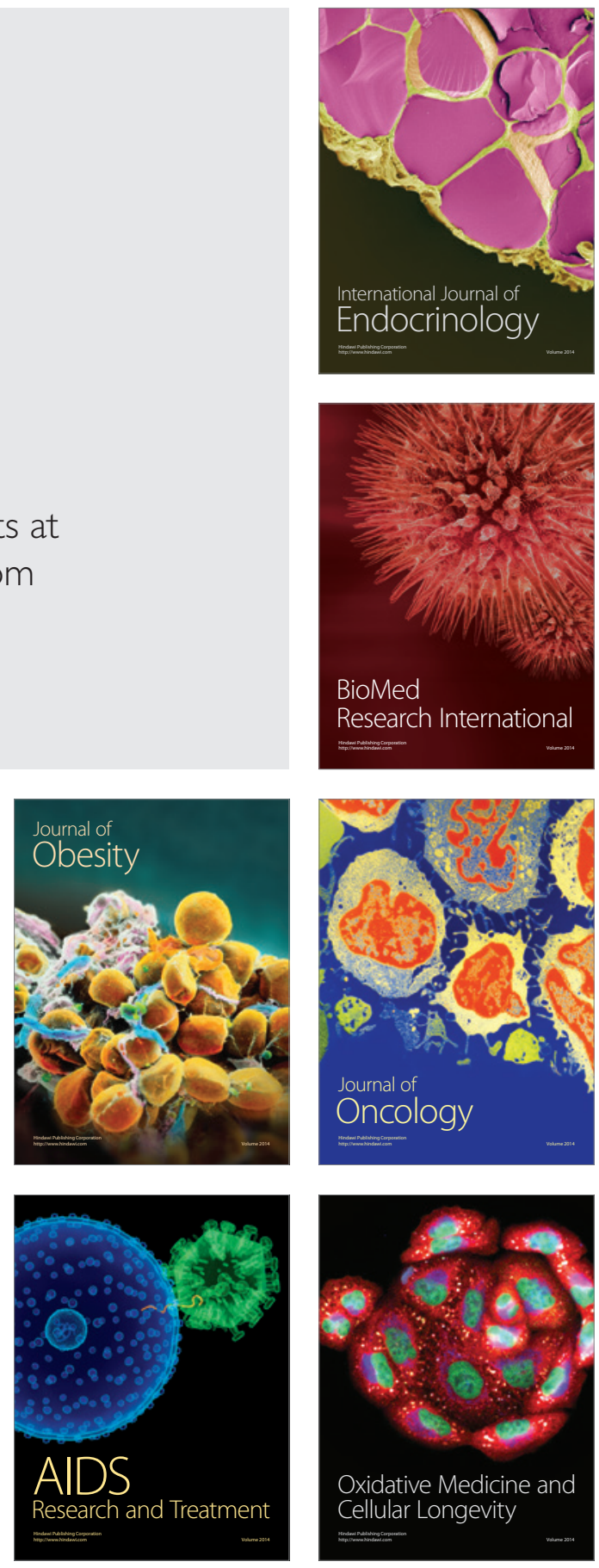